\title{
A Long-term survival due to repeated surgical resections for recurrent retroperitoneal liposarcoma
}

\author{
Mai Shiga $^{1)}$, Hiromichi Maeda $^{2)}$, Ken Okamoto $^{2)}$, Michiya Kobayashi ${ }^{2)}$, Kazuhiro Hanazaki $^{1)}$ \\ 1) Department of Surgery, Kochi Medical School, Kochi University \\ ${ }^{2)}$ Cancer Treatment Center, Kochi Medical School Hospital
}

\begin{abstract}
Background: Surgical resection is the mainstay of treatment for retroperitoneal liposarcoma; however, local recurrence is frequent and treatments for such cases remain controversial.

Results: A 63-year-old female presented at our hospital after two weeks of fever and showing a palpable mass in the left abdomen. A large, low-density mass on enhanced computed tomography was diagnostic for retroperitoneal liposarcoma, and the patients underwent tumor resection with left kidney. From that time, the patient underwent 13 surgical resections over 11 years, including the first surgery, with multiple visceral organ resections for retroperitoneal liposarcoma and its recurrence. Conclusion: Repeated surgeries could prolong survival and postpone the emergence of tumor-related symptoms in patients with recurrent retroperitoneal liposarcoma.
\end{abstract}

Keywords: retroperitoneal, liposarcoma, surgery

(Received: July 25, 2017; Accepted August 10, 2017)

\section{Introduction}

Retroperitoneal liposarcoma is the most common type of soft tissue sarcoma arising from the retroperitoneal space $^{1)}$. Complete resection with negative microscopic margins is the aim for disease cure; however, this approach is often hampered by ambiguous macroscopic margins and the involvement of vital organs such as pancreas, kidney, adrenal gland, and major blood vessels ${ }^{2)}$. Consequently, tumor recurrence is often encountered near the surgical field and at distant sites within the abdominal cavity. In such cases, local control of the tumor can be achieved by surgical resection or radiological modalities, while systemic chemotherapy or palliative medication could be provided for tumor-related symptoms. Overall, the best strategy for treating or controlling retroperitoneal liposarcoma remains undetermined, mainly due to the rarity, heterogeneous presentation, and biological nature of the tumors.

Herein we report a patient with retroperitoneal liposarcoma. The patient had multiple recurrences within the abdominal cavity and surgical resection was performed 13 times during the 11 years of treatment.

Corresponding Author: Hiromichi Maeda, MD, PhD. Cancer Treatment Center Kochi Medical School Hospital, Kochi University, Kohasu, Oko-cho, Nankoku, Kochi, 783-8505, Japan Telephone: +81-88-880-2370, Fax:+81-88-880-2371, E-mail hmaeda@kochi-u.ac.jp

\section{Case presentation}

Initially, a 63-year-old female presented at hospital following two weeks of fever. Computed tomography (CT) to investigate a palpable abdominal mass confirmed a low-density area in the left abdomen encapsulating the left kidney (Fig. 1). The patient underwent tumor resection with left kidney under the diagnosis of retroperitoneal liposarcoma. Pathologically the tumor comprised vacuolated adipocytes of different sizes (Fig. 1), and infiltration of the tumor cells to the kidney was observed, leading to a final diagnosis of de-differentiated liposarcoma of the retroperitoneum. Afterwards, the patient visited hospital every three to six month and fluorodeoxyglucose-positron emission tomography-CT (FDG-PET$\mathrm{CT}$ ) or enhanced CT was obtained at intervals of four to six months.

At 39 months after the first operation, local recurrence of the tumor was detected at the dorsal side of the pancreatic tail (Fig. 2), resulting in surgical resection of the affected area. Five months later, further tumor recurrence was identified near the left psoas muscle and again, surgically resected. Subsequently, this patient experienced 10 separate tumor recurrences, each of which were treated by surgical resection. The tumor was consistently diagnosed as dedifferentiated liposarcoma from the initial diagnosis to the last treatment. 

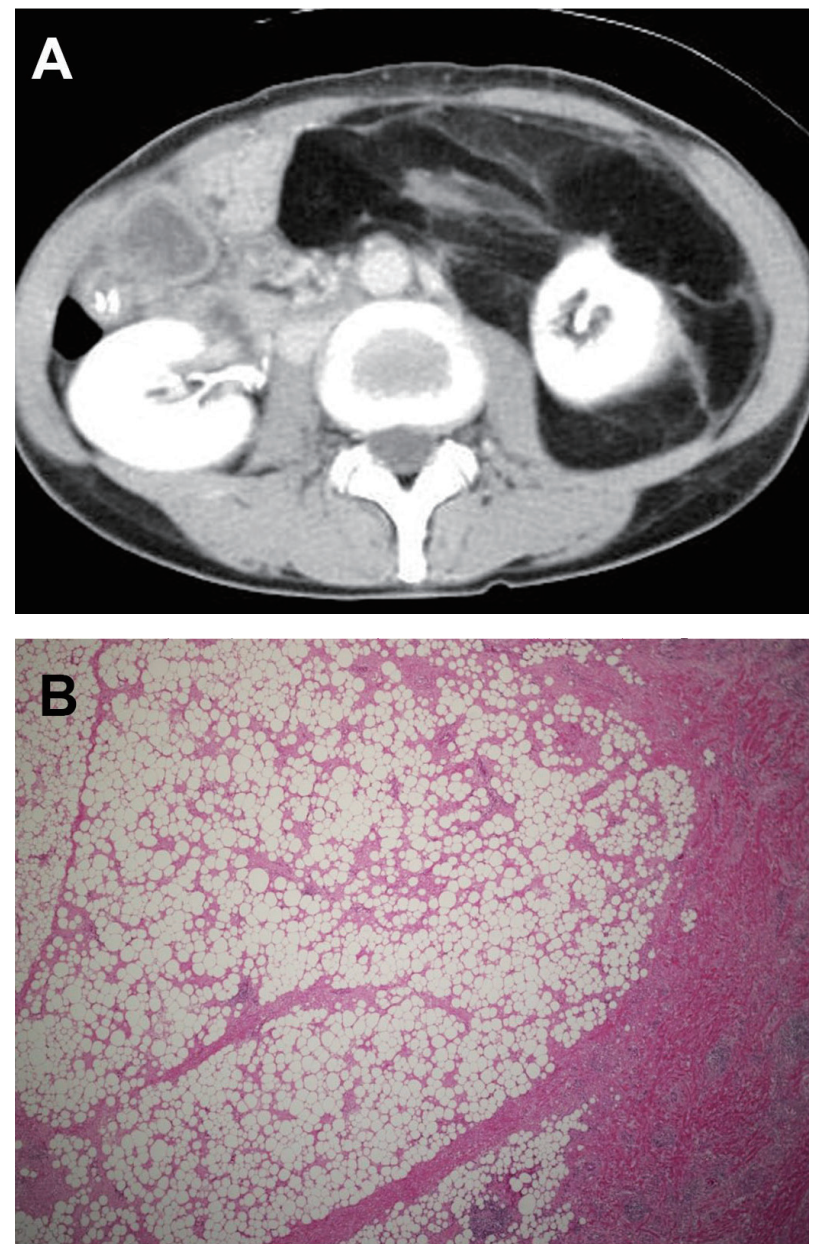

Fig. 1 Enhanced computed tomography and pathological examination for the first and second operation

(A) A low density mass was observed in the left upper abdomen surrounding the left kidney.

(B) HE staining of the resected specimen showing the vacuolated adipocytes of different sizes, resulting in the diagnosis of liposarcoma.

At 11 years after the initial diagnosis, the patient had another tumor recurrence near the posterior wall of the stomach, the transverse colon, and adjacent to the aorta (Fig. 3A). The patient underwent their $13^{\text {th }}$ tumor resection with partial resection of transverse colon and wedge resection of the gastric posterior wall; however, the tumor mass adjacent to the abdominal aorta could not be removed due to strong adhesion between tumor and aorta. Her postoperative course was further complicated by leakage from the closure of the stomach, and rapid growth of tumor was identified on abdominal CT at 12 weeks after the surgery (Fig. 3B). The patient died three months after the last operation, and 11 years after the initial diagnosis of her retroperitoneal liposarcoma.

\section{Discussion}

Liposarcoma is the most common type (approximately
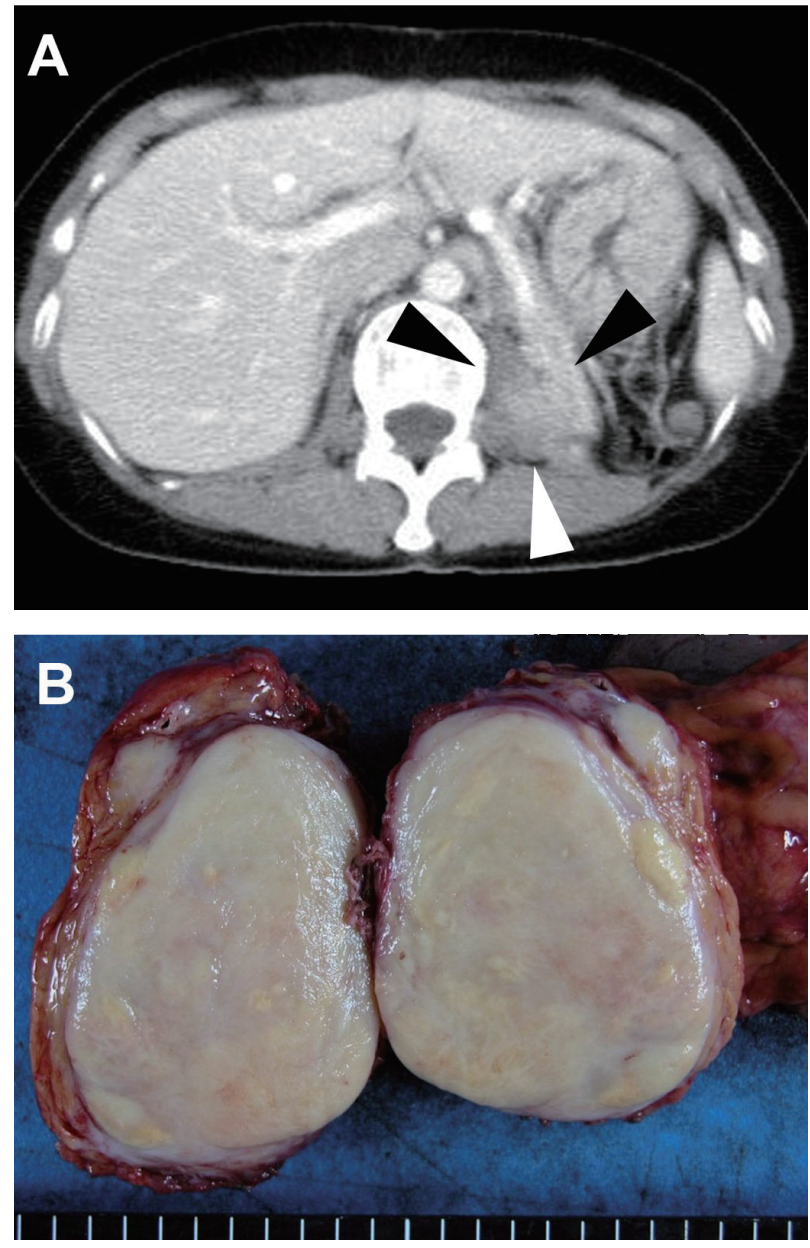

Fig. 2 Abdominal computed tomography and resected specimen at second operation

(A) CT examination performed 39 months after the first operation showing recurrence of the liposarcoma dorsal to the pancreatic body and tail (arrowhead).

(B) Macroscopically, the resected specimen showed a solid whitish tumor with expansive growth.

$40 \%$ ) of retroperitoneal soft tissue sarcomas ${ }^{1,3)}$, although due to the overall rarity of soft tissue sarcoma, liposarcoma is often described and analyzed together with other sarcoma types. However, unlike other sarcomas, it is generally difficult to identify the margin between normal adipose tissue and liposarcomas, which are also more frequently accompanied by local recurrence ${ }^{3)}$ and resistance to chemotherapy ${ }^{4}$. Therefore, future clinical studies need to establish a management strategy for retroperitoneal liposarcoma, separately from other types of sarcoma.

The 5-year overall survival for patients with retroperitoneal liposarcoma is approximately $50 \%{ }^{5)}$, although controversy remains as to whether the prognosis of retroperitoneal liposarcoma is more dismal than that for other histological types of sarcoma. The most significant factor determining a poor prognosis is positive surgical margins, and surgical intervention for volume reduction only 

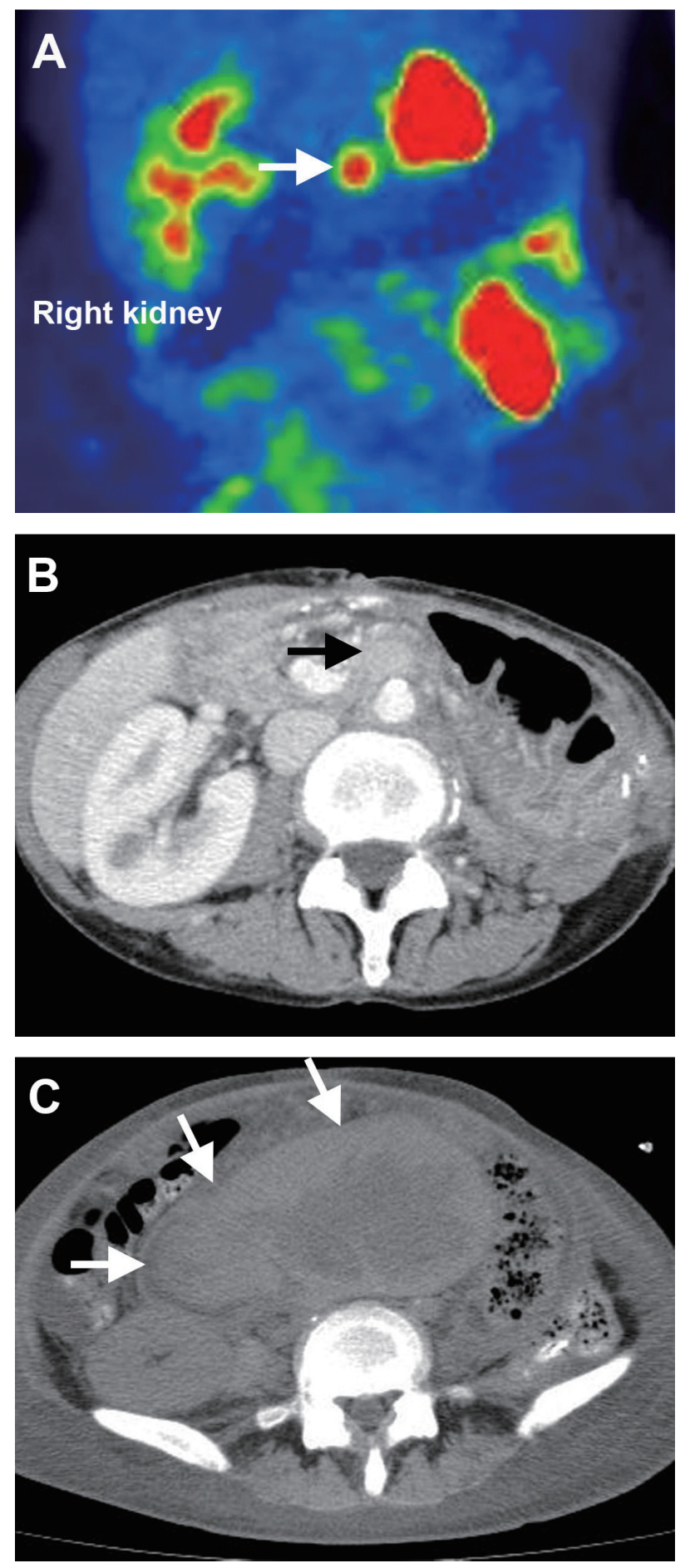

Fig. 3 Abdominal computed tomography at $13^{\text {th }}$ operation

(A) FDG-PET-CT demonstrated strong accumulation of FDG in the left upper and lower abdomen. The white arrow indicates a nodule located immediately ventral to the abdominal aorta.

(B) Appearance of the nodule identified on CT before operation.

(C) Rapidly enlarged tumor at 12 weeks after surgery showing the tumor compressing the aorta (arrowhead indicates the tumor). provides no survival benefit over non-surgical treatment ${ }^{5)}$, although it might alleviate tumor-related symptoms ${ }^{6}$. In the present case, a macroscopic margin-negative resection was achieved in all but the $13^{\text {th }}$ and last surgical resection, probably contributing to the long-term survival, more than 10 year despite multiple recurrence.

Distant metastasis of liposarcoma is common when they are large in size, of de-differentiated histological type, grade 2-3, stage II-III, and margin positive ${ }^{7)}$. In the present case, distant metastasis was not observed despite its size, repetitive local recurrence within the abdominal cavity and pathological subtype, which could have contributed to the patient's long-term survival. We should also note that frequent hospital visits and periodical radiological examination could facilitate detecting the resectable recurrences and thus lead to a better outcome. In particular, FDG-PET-CT was useful to detect small liposarcomas within the intra-abdominal scars, which were due to the multiple operations. Although the efficacy of FDG-PET-CT to detect recurrent de-differentiated liposarcoma is not clinically established, we believe that this modality is useful both in screening for recurrence and for preoperative evaluation of other potential metastatic sites.

So far and to our knowledge, this is the first case report describing multiple surgical resections of recurrent retroperitoneal liposarcoma, leading to the survival more than 10 years. Because this is a single experience of favorable outcome, definite conclusion should not be drawn; more reports including unfavorable results should be accumulated to establish an optimal treatment strategy for this rare disease.

In conclusion, we experienced a patient with retroperitoneal de-differentiated liposarcoma. We consider that repeated resections of the recurrent liposarcoma over many years prolonged survival and prevented the emergence of tumor-related symptoms in this selected patient.

\section{Informed consent}

The patient gave permission for publication of this report.

\section{References}

1) Lewis JJ, Leung D, Woodruff JM, Brennan MF. Retroperitoneal soft-tissue sarcoma: analysis of 500 patients treated and followed at a single institution. Ann Surg. 1998; 228: 355-65.

2) Messiou C, Moskovic E, Vanel D, Morosi C, Benchimol R, Strauss D, Miah A, Douis H, van Houdt W, Bonvalot S. Primary retroperitoneal soft tissue sarcoma: Imaging appearances, pitfalls and diagnostic algorithm. Eur J Surg Oncol. 2017; 43: 1191-1198.

3) Abdelfatah E, Guzzetta AA, Nagarajan N, Wolfgang CL, Pawlik TM, Choti MA, Schulick R, Montgomery EA, Meyer C, Thornton K, Herman J, Terezakis S, Frassica D, Ahuja N. Long-term outcomes in treatment of retroperitoneal sarcomas: A 15 year singleinstitution evaluation of prognostic features. J Surg Oncol. 2016; 114: 56-64. 
4) Abbas Manji G, Singer S, Koff A, Schwartz GK. Application of molecular biology to individualize therapy for patients with liposarcoma. Am Soc Clin Oncol Educ Book. 2015:213-8.

5) Lee SY, Goh BK, Teo MC, Chew MH, Chow PK, Wong WK, Ooi LL, Soo KC. Retroperitoneal liposarcomas: the experience of a tertiary Asian center. World J Surg Oncol. 2011; 9: 12.
6) Shibata D, Lewis JJ, Leung DH, Brennan MF. Is there a role for incomplete resection in the management of retroperitoneal liposarcomas? J Am Coll Surg. 2001; 193: 373-9.

7) Singer S, Antonescu CR, Riedel E, Brennan MF. Histologic subtype and margin of resection predict pattern of recurrence and survival for retroperitoneal liposarcoma. Ann Surg. 2003; 238: 358-70; discussion 370-1. 